\title{
Reductive removal of the Boc protecting group via a DTBB-catalysed lithiation reaction
}

\author{
Raquel Almansa, Cherif Behloul, David Guijarro, and Miguel Yus* \\ Departamento de Química Orgánica, Facultad de Ciencias and Instituto de Síntesis Orgánica \\ (ISO), Universidad de Alicante, Apdo. 99, 03080 Alicante (Spain) \\ E-mail:yus@ua.es
}

\section{Dedicated to Professor Atta-ur-Rahman on occasion of his $65^{\text {th }}$ birthday}

\begin{abstract}
The DTBB-catalysed lithiation of Boc-protected alcohols, amines and thiols in THF at $0^{\circ} \mathrm{C}$ led, after quenching with methanol or water, to the recovery of the free alcohols, amines and thiols in short reaction times and in moderate to very good yields. The procedure has been applied to primary, secondary and tertiary alcohols, phenols, secondary amines and primary, secondary and aromatic thiols. This method represents a reasonable alternative to the previously reported deprotection procedures.
\end{abstract}

Keywords: Lithium, DTBB, tert-butoxycarbonyl, Boc, deprotection, alcohols, amines, thiols

\section{Introduction}

The protection of a functional group can be essential in the chemistry of polyfunctionalised molecules when a reaction has to be carried out in a part of the compound without perturbing the rest of the molecule. In the case of compounds of the type RYH for Y $=\mathrm{O}, \mathrm{NR}$, S, especially for amines, among the different possibilities, the tert-butoxycarbonyl protecting group (Boc; introduced in $1957^{1}$ ) has demonstrated to be one of the most efficient ones. ${ }^{2,3,4}$ Actually, the Boc group is the most frequently used as amino-protecting functionality in peptide chemistry. Reasons for the mentioned success of the Boc group are the classical properties for a protecting group: (a) It is easily introduced using commercially available di-tert-butyl dicarbonate [also called tert-butyl pyrocarbonate: $\left.\left(\mathrm{Bu}^{\mathrm{t} O C O}\right)_{2} \mathrm{O}\right]$ under standard basic conditions (sodium hydroxide, aqueous dioxane); (b) It is stable towards bases, as well as to catalytic hydrogenation and reduction with sodium in liquid ammonia; and (c) Its removal can be easily achieved under

\footnotetext{
*Corresponding author. Fax: +34-965903549; e-mail: yus@ua.es
} 
acidic conditions [hydrochloric acid (in dichloromethane, ether or ethyl acetate) or trifluoroacetic acid (neat or in dichloromethane)]. In especial cases, more sophisticated procedures can be used for both, the introduction of the Boc group [with tert-butyl chloro- or fluoroformate, tert-butyl azidoformate, or 2-(tert-butoxycarbonyloxyimino)-2-phenylacetonitrile] and its cleavage (trimethylsilyl $^{5}$ or tert-butyldimethylsilyl triflate, ${ }^{6}$ boron trifluoride etherate, ${ }^{7}$ tetrabutylammonium fluoride, ${ }^{8}$ zinc dibromide, ${ }^{9}$ or cerium ammonium nitrate ${ }^{10}$ ). The generally accepted mechanism for the cleavage of the Boc group under acidic conditions involves the formation of carbon dioxide and the tert-butyl cation, which after loosing a proton gives isobutene, so only volatile products are generated together with the desired deprotected product making the work-up of the reaction very convenient.

On the other hand, in the last few years we have been using an arene-catalysed lithiation to perform metallations under very mild reaction conditions. ${ }^{11,12,13}$ Among other uses, ${ }^{14}$ this methodology has been shown to be applicable to the cleavage of trityl ethers ${ }^{15}$ and amines, ${ }^{16}$ to the desilylation ${ }^{17}$ and the deallyloxy- or debenzyloxycarbonylation ${ }^{18}$ of protected alcohols, amines and thiols, and to perform the deacylation of esters, thioesters and amides. ${ }^{19}$ In this paper we report on the reductive removal of the Boc group from protected alcohols, amines and thiols via a DTBB-catalysed lithiation process under very mild reaction conditions. ${ }^{20}$

\section{Results and Discussion}

The reaction of $O$-Boc protected alcohols 1a-d or protected phenol 1e with an excess of lithium powder (1:9 molar ratio) and a catalytic amount of 4,4'-di-tert-butylbiphenyl (DTBB; 1:0.1 molar ratio; $5 \mathrm{~mol} \%$ ) in THF at $0^{\circ} \mathrm{C}$ and for $1-3 \mathrm{~h}$ led, after quenching with methanol, to the corresponding alcohols 2a-d or phenol 2e, respectively (Scheme 1 and Table 1, entries 1, 3, 4, 5 and 7). Yields of the deprotected products were very good, except when R was a tertiary alkyl or a phenyl group (Table 1, entries 5 and 7). In the latter two cases, there was some unreacted starting material at the end of the reactions. So, the lithiation reactions were repeated and the stirring was maintained for $7 \mathrm{~h}$ at $0^{\circ} \mathrm{C}$. Since the starting material did not react further at that temperature, the reactions were stirred at room temperature for $13 \mathrm{~h}$ (for compound $\mathbf{1 d}$ ) or $17 \mathrm{~h}$ (for compound 1e). Carbonate 1d disappeared completely under these conditions and the yield of the deprotected alcohol 2d was improved up to 69\% (Table 1, entry 6). However, carbonate 1e did not react completely after $17 \mathrm{~h}$ at room temperature, although the yield of the phenol $2 \mathrm{e}$ was higher than the one obtained in the first experiment (compare entries 7 and 8 in Table 1). We think that the lower yields obtained in the deprotection of compounds $\mathbf{1 d}$ and $\mathbf{1 e}$ could be due to competition between the cleavage of the R-oxygen bond and the tert-butyl-oxygen bond. A proof for this competition is the fact that the hydrocarbons resulting from the cleavage of the R-oxygen bond (2,6-dimethyloctane from 1d and mesitylene from 1e) were detected (GC-MS) in the crude reaction mixtures. 


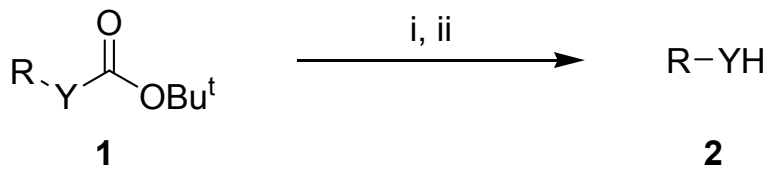

Scheme 1. Reagents and conditions: (i) Li, DTBB (5 mol\%), THF, $0^{\circ} \mathrm{C}$; (ii) $\mathrm{MeOH}$ or $\mathrm{H}_{2} \mathrm{O}$.

In order to evaluate the potential of our deprotection procedure, we repeated the lithiation of carbonate 1a on a $10 \mathrm{mmol}$ scale. The amount of lithium was reduced to a $1 \mathrm{a} /$ lithium molar ratio of 1:4 and the reactions were performed with a higher concentration of the substrate. After filtration to remove the excess of lithium powder and hydrolysis with water, pure 1-decanol was isolated in $82 \%$ yield after work-up and purification by column chromatography (Table 1, entry 2 ). This result shows the synthetic usefulness of our deblocking procedure.

The same deprotection procedure was applied to the carbamates $\mathbf{1 f}-\mathbf{h}$ and the expected amines 2f-h were obtained in good to excellent yields (Scheme 1 and Table 1, entries 9-13). We also tried to remove the Boc group from a protected primary amine, but it failed. The deprotection of $N$-(tert-butoxycarbonyl)octylamine was attempted following the same procedure previously used by us in the deprotection of tritylated primary amines, ${ }^{16}$ consisting in a deprotonation with $n$-butyllithium and treatment with trimethylsilyl chloride before performing the lithiation step. Although the starting material disappeared, neither octylamine nor $N$ (trimethylsilyl)octylamine were detected in the crude reaction mixture (GC-MS). The lithiation of the $S$-Boc protected thiols $\mathbf{1} \mathbf{i}-\mathbf{k}$ gave only moderate yields of the thiols $\mathbf{2} \mathbf{i}-\mathbf{k}$ (Table 1 , entries 14-16), probably due to oxidation of the latter to the corresponding disulfides by the air after the reaction had been quenched with methanol. These disulfides were detected in the crude reaction mixtures (GC-MS).

The reductive cleavage of starting materials having an allylic or benzylic $\mathrm{R}$ group was also attempted. Benzyl tert-butyl carbonate, tert-butyl geranyl carbonate, $N$-(tert-butoxycarbonyl)- $N$ methylbenzylamine and $N$-(tert-butoxycarbonyl)diallylamine were submitted to the lithiation process, but the desired deprotected alcohols or amines were not formed. The reductive cleavage of the allylic or benzylic carbon-heteroatom bond was the preferred reaction pathway in these cases, leading to the formation of the corresponding lithium carbonates or $N$-lithiocarbamates and benzyl-, allyl- or geranyllithium. The generated allylic and benzylic organolithium compounds would give the corresponding hydrocarbons on reaction with methanol. Toluene was detected (GC-MS) in the crude of the reactions with the benzylic substrates, which would confirm the cleavage of the benzylic carbon-heteroatom bond. 
Table 1. Reductive deprotection of compounds 1 via a DTBB-catalysed lithiation. Preparation of compounds 2

\begin{tabular}{|c|c|c|c|c|c|c|}
\hline \multirow[b]{2}{*}{ Entry } & \multicolumn{3}{|c|}{ Substrate } & \multirow[b]{2}{*}{ Time (h) } & \multicolumn{2}{|c|}{ Product } \\
\hline & No. & $\mathrm{Y}$ & $\mathrm{R}$ & & No. & Yield $(\%)^{\mathrm{a}, \mathrm{b}}$ \\
\hline 1 & $1 \mathbf{a}$ & $\mathrm{O}$ & $\mathrm{Me}\left(\mathrm{CH}_{2}\right)_{9}$ & 1.5 & $2 \mathbf{a}$ & $>99^{c}$ \\
\hline 2 & $1 \mathbf{a}$ & $\mathrm{O}$ & $\mathrm{Me}\left(\mathrm{CH}_{2}\right)_{9}$ & 5.0 & $2 \mathbf{a}$ & --- $(82)^{\mathrm{d}, \mathrm{e}}$ \\
\hline 3 & $1 b$ & $\mathrm{O}$ & $\mathrm{Me}\left(\mathrm{CH}_{2}\right)_{5} \mathrm{CH}(\mathrm{Me})$ & 3.0 & $2 \mathbf{b}$ & $70^{\mathrm{c}}$ \\
\hline 4 & 1c & $\mathrm{O}$ & $c-\mathrm{C}_{6} \mathrm{H}_{11}$ & 3.0 & $2 c$ & $70^{\mathrm{c}}$ \\
\hline 5 & $1 d$ & $\mathrm{O}$ & $\operatorname{Pr}^{\mathrm{i}}\left(\mathrm{CH}_{2}\right)_{3} \mathrm{C}(\mathrm{Me})(\mathrm{Et})$ & 1.0 & $2 d$ & $45^{\mathrm{c}}$ \\
\hline 6 & $1 d$ & $\mathrm{O}$ & $\operatorname{Pr}^{\mathrm{i}}\left(\mathrm{CH}_{2}\right)_{3} \mathrm{C}(\mathrm{Me})(\mathrm{Et})$ & $20.0^{\mathrm{f}}$ & $2 d$ & $69(42)^{\mathrm{e}}$ \\
\hline 7 & $1 \mathrm{e}$ & $\mathrm{O}$ & $2,4,6-\mathrm{Me}_{3} \mathrm{C}_{6} \mathrm{H}_{2}$ & 2.5 & $2 e$ & $25^{\mathrm{c}}$ \\
\hline 8 & $1 e$ & $\mathrm{O}$ & $2,4,6-\mathrm{Me}_{3} \mathrm{C}_{6} \mathrm{H}_{2}$ & $24.0^{\mathrm{g}}$ & $2 \mathbf{e}$ & $44(30)^{\mathrm{e}}$ \\
\hline 9 & $1 f$ & $\mathrm{Me}\left(\mathrm{CH}_{2}\right)_{7} \mathrm{~N}$ & $\mathrm{Me}\left(\mathrm{CH}_{2}\right)_{7}$ & 1.5 & $2 f$ & $>99^{c}$ \\
\hline 10 & $1 \mathrm{~g}$ & $--{ }^{h}$ & $-{ }^{h}$ & 2.0 & $2 \mathrm{~g}^{\mathrm{i}}$ & $>99^{c}$ \\
\hline 11 & $1 \mathrm{~g}$ & $-{ }^{h}$ & $-{ }_{--}^{h}$ & 3.0 & $2 \mathrm{~g}^{\mathrm{i}}$ & $89(81)^{\mathrm{e}}$ \\
\hline 12 & $1 \mathrm{~h}$ & $\mathrm{MeN}$ & $\mathrm{Ph}$ & 2.0 & $2 h$ & $62^{c}$ \\
\hline 13 & $1 \mathrm{~h}$ & $\mathrm{MeN}$ & $\mathrm{Ph}$ & 2.0 & $2 \mathrm{~h}$ & $55(49)^{\mathrm{e}}$ \\
\hline 14 & $\mathbf{1 i}$ & $\mathrm{S}$ & $\mathrm{Me}\left(\mathrm{CH}_{2}\right)_{9}$ & 5.0 & $2 \mathbf{i}$ & $47^{\mathrm{c}}$ \\
\hline 15 & $1 \mathbf{j}$ & $\mathrm{S}$ & $c-\mathrm{C}_{6} \mathrm{H}_{11}$ & 3.0 & $2 \mathrm{j}$ & $20^{\mathrm{c}}$ \\
\hline 16 & $1 \mathrm{k}$ & $\mathrm{S}$ & $\mathrm{Ph}$ & 1.5 & $2 \mathbf{k}$ & $39^{c}$ \\
\hline
\end{tabular}

${ }^{\text {a }}$ Yield determined by quantitative GLC, using commercially available compounds $\mathbf{2}, n$-dodecane (internal standard) and $n$-hexadecane (internal standard for $\mathbf{2 e}$ ) in the determination of response factors.

${ }^{\mathrm{b}}$ In brackets, isolated yield (see experimental part). All isolated compounds 2 were $>95 \%$ pure (300 $\mathrm{MHz}{ }^{1} \mathrm{H}$ NMR and/or GLC).

${ }^{\mathrm{c}}$ The reaction was quenched with methanol.

${ }^{d}$ The reaction was performed on a $10 \mathrm{mmol}$ scale.

${ }^{\mathrm{e}}$ The reaction was hydrolysed with water. 
${ }^{\mathrm{f}}$ The reaction was stirred at $0^{\circ} \mathrm{C}$ for $7 \mathrm{~h}$ and at room temperature for $13 \mathrm{~h}$.

${ }^{g}$ The reaction was stirred at $0^{\circ} \mathrm{C}$ for $7 \mathrm{~h}$ and at room temperature for $17 \mathrm{~h}$.

${ }^{\mathrm{h}} \mathbf{1 g}=4$-benzyl- $N$-(tert-butoxycarbonyl)piperidine.

${ }^{\mathrm{i}} \mathbf{2 g}=4$-benzylpiperidine.

Concerning a possible reaction mechanism, we assume that the reductive cleavage of the tert-butyl-oxygen bond takes place first, leading to the tert-butyl radical and the corresponding lithium carbonate (for 1a-e), carbamate (for 1f-h) or thiocarbonate (for 1i-k). The latter three species would then decarboxylate giving the lithium salts of the alcohols $\mathbf{2 a - d}$, the phenol $\mathbf{2 e}$, the amines $\mathbf{2} \mathbf{f}-\mathbf{h}$ or the thiols $\mathbf{2 i}-\mathbf{k}$, respectively, which would yield the final products after protonolysis by methanol or water. The tert-butyl radical could deproportionate to give isobutane and isobutene. These two by-products and the carbon dioxide generated during the decarboxylation step are volatile and are easily separated from the desired reaction products.

Starting materials $\mathbf{1}$ were prepared from commercially available alcohols $\mathbf{2 a - d}$, phenol $\mathbf{2 e}$, amines $\mathbf{2 f - h}$ and thiols $\mathbf{2 i - k}$ by reaction with di-tert-butyl dicarbonate and a catalytic amount of 4-(dimethylamino)pyridine (DMAP; 1:0.4 molar ratio) in toluene at room temperature.

\section{Conclusions}

In conclusion, from the results described here, we think that the reductive deprotection of Bocprotected alcohols, amines and thiols by a DTBB-catalysed lithiation procedure represents a reasonable alternative to other deprotection techniques, being of especial interest taking into account that the same protected substrates are inert towards sodium in liquid ammonia (see the Introduction section).

\section{Experimental}

General Procedures. For general experimental information, see reference 16. All reagents used for the synthesis of Boc-protected substrates 1 and DTBB were commercially available (Acros, Aldrich) and were used without further purification. Lithium powder was prepared according to the procedure described in reference 21 . Commercially available $n$-butyllithium was titrated with a $1 \mathrm{M}$ solution of sec-butanol in xylene using 1,10-phenanthroline as indicator. ${ }^{22}$ Commercially available toluene (Merck) and anhydrous THF (99.9\%, water content $\leq 0.006 \%$, Acros) were used as solvents in the reactions. All glassware was dried in an oven at $100^{\circ} \mathrm{C}$ and cooled to room temperature under Ar before use.

Preparation of the starting Boc-protected materials 1. General procedure. 4(Dimethylamino)pyridine ( $96 \mathrm{mg}, 0.4 \mathrm{mmol}$ ) was added in one portion to a stirred solution of the corresponding alcohol, amine or thiol $1(10.0 \mathrm{mmol})$ and di-tert-butyl dicarbonate $(2.18 \mathrm{~g}, 10.0$ 
$\mathrm{mmol})$ in toluene $(15 \mathrm{~mL})$ at room temperature. After stirring for $30 \mathrm{~min}$ at the same temperature, the crude reaction mixture was adsorbed on basic aluminium oxide, transferred to a short column of basic aluminium oxide and eluted with hexane. Evaporation of the solvent (15 Torr) afforded the expected Boc-protected compounds $\mathbf{1}$ in pure form. Their corresponding physical, spectroscopic and analytical data follow.

tert-Butyl 1-decyl carbonate (1a). ${ }^{23}$ Colourless oil; yield: 54\%; $R_{\mathrm{f}} 0.96$ (hexane/ethyl acetate: 9/1); v (film) $1743(\mathrm{C}=\mathrm{O}), 1279,1255 \mathrm{~cm}^{-1}(\mathrm{CO}) ; \delta_{\mathrm{H}} 0.88\left(3 \mathrm{H}, \mathrm{t}, J=6.8 \mathrm{~Hz}, \mathrm{MeCH}_{2}\right), 1.14-1.41$ [14H, m, $\left.\mathrm{Me}\left(\mathrm{CH}_{2}\right)_{7}\right], 1.49\left(9 \mathrm{H}, \mathrm{s}, \mathrm{Me}_{3} \mathrm{C}\right), 1.58-1.73\left(2 \mathrm{H}, \mathrm{m}, \mathrm{CH}_{2} \mathrm{CO}\right), 4.05(2 \mathrm{H}, \mathrm{t}, J=6.8 \mathrm{~Hz}$, $\left.\mathrm{CH}_{2} \mathrm{O}\right) ; \delta_{\mathrm{C}} 14.1(\mathrm{Me}), 22.6,25.7,28.7,29.2,29.25,29.3,29.5,31.9\left[\mathrm{Me}\left(\mathrm{CH}_{2}\right)_{8}\right], 27.7(3 \mathrm{C}$, $\left.\mathrm{Me}_{3} \mathrm{C}\right), 67.2\left(\mathrm{CH}_{2} \mathrm{O}\right), 81.6(\mathrm{CO}), 153.7(\mathrm{C}=\mathrm{O}) ; \mathrm{m} / z 258\left(\mathrm{M}^{+},<1 \%\right), 141$ (71), 140 (44), 112 (27), 111 (27), 99 (28), 98 (17), 97 (43), 85 (100), 84 (27), 83 (53), 82 (20), 71 (84), 70 (41), 69 (49), 68 (14), 67 (10), 57 (89), 56 (34), 55 (56).

tert-Butyl 2-octyl carbonate (1b). Colourless oil; yield: 64\%; $R_{\mathrm{f}} 0.96$ (hexane/ethyl acetate: 9/1); $v$ (film) $1737(\mathrm{C}=\mathrm{O}), 1264 \mathrm{~cm}^{-1}(\mathrm{CO}) ; \delta_{\mathrm{H}} 0.88\left(3 \mathrm{H}, \mathrm{t}, J=6.9 \mathrm{~Hz}, \mathrm{MeCH}_{2}\right), 1.14-1.74(13 \mathrm{H}$, $\mathrm{m}, 5 \times \mathrm{CH}_{2}$ and $\left.\mathrm{MeCH}\right), 1.48\left(9 \mathrm{H}, \mathrm{s}, \mathrm{Me}_{3} \mathrm{C}\right), 4.62-4.82(1 \mathrm{H}, \mathrm{m}, \mathrm{CHO}) ; \delta_{\mathrm{C}} 14.0\left(\mathrm{MeCH}_{2}\right), 19.9$ $(\mathrm{MeCH}), 21.4,25.3,27.8,29.1,31.7\left(5 \times \mathrm{CH}_{2}\right), 22.5\left(3 \mathrm{C}, \mathrm{Me}_{3} \mathrm{C}\right), 74.1(\mathrm{CHO}), 81.4(\mathrm{CO}), 153.3$ $(\mathrm{C}=\mathrm{O}) ; m / z 173\left(\mathrm{M}^{+}-57,<1 \%\right), 113$ (57), 112 (52), 84 (11), 83 (18), 71 (100), 70 (21), 69 (17), 57 (77), 56 (15), 55 (22); HRMS: $\mathrm{M}^{+}-57$, found 173.1171. $\mathrm{C}_{9} \mathrm{H}_{17} \mathrm{O}_{3}$ requires 173.1200.

tert-Butyl cyclohexyl carbonate (1c). ${ }^{24}$ Colourless oil; yield: $73 \% ; R_{\mathrm{f}} 0.96$ (hexane/ethyl acetate: $9 / 1) ; v($ film $) 1736(\mathrm{C}=\mathrm{O}), 1281,1254 \mathrm{~cm}^{-1}(\mathrm{CO}) ; \delta_{\mathrm{H}} 1.08-2.05\left(10 \mathrm{H}, \mathrm{m}, 5 \times \mathrm{CH}_{2}\right), 1.49$ $\left(9 \mathrm{H}, \mathrm{s}, \mathrm{Me}_{3} \mathrm{C}\right), 4.43-4.66(1 \mathrm{H}, \mathrm{m}, \mathrm{CH}) ; \delta_{\mathrm{C}} 23.8(2 \mathrm{C}), 23.95(2 \mathrm{C}), 25.2\left(5 \times \mathrm{CH}_{2}\right), 27.8\left(3 \mathrm{C}, \mathrm{Me}_{3} \mathrm{C}\right)$, $76.4(\mathrm{CHO}), 81.6(\mathrm{CO}), 153.1(\mathrm{C}=\mathrm{O}) ; \mathrm{m} / z 143\left(\mathrm{M}^{+}-57,<1 \%\right), 83$ (39), 82 (17), 67 (10), 59 (14), 57 (100), 55 (16).

tert-Butyl 3,7-dimethyl-3-octyl carbonate (1d). Colourless oil; yield: 32\%; $R_{\mathrm{f}} 0.71$ (hexane/ethyl acetate: 9/1); $v\left(\right.$ film) $1739(\mathrm{C}=\mathrm{O}), 1290 \mathrm{~cm}^{-1}(\mathrm{CO}) ; \delta_{\mathrm{H}} 0.88,0.89(6 \mathrm{H}, 2 \mathrm{~d}, J=6.4$, $6.6 \mathrm{~Hz}$, respectively, $\left.\mathrm{Me}_{2} \mathrm{CH}\right), 1.08-2.06\left(12 \mathrm{H}, \mathrm{m}, \mathrm{MeCH}_{2}, \mathrm{CH}\right.$ and $\left.4 \times \mathrm{CH}_{2}\right), 1.38(3 \mathrm{H}, \mathrm{s}$, $\left.M e \mathrm{CCH}_{2}\right), 1.47\left(9 \mathrm{H}, \mathrm{s}, \mathrm{Me}_{3} \mathrm{C}\right) ; \delta_{\mathrm{C}} 8.2\left(\mathrm{MeCH}_{2}\right), 22.5\left(2 \mathrm{C}, \mathrm{Me}_{2} \mathrm{CH}\right), 22.6\left(\mathrm{MeCCH}_{2}\right), 23.2,30.7$, 39.2, $39.3\left(4 \times \mathrm{CH}_{2}\right), 27.85\left(3 \mathrm{C}, \mathrm{Me}_{3} \mathrm{C}\right), 80.9\left(\mathrm{CMe}_{3}\right), 85.7(\mathrm{EtCO}), 152.0(\mathrm{C}=\mathrm{O}) ; \mathrm{m} / \mathrm{z} 141\left(\mathrm{M}^{+}-\right.$ 117, 15\%), 140 (27), 129 (16), 111 (15), 85 (10), 83 (16), 73 (32), 71 (16), 70 (58), 69 (43), 59 (31), 57 (100), 56 (25), 55 (53); HRMS: $\mathrm{M}^{+}-117$, found 141.1646. $\mathrm{C}_{10} \mathrm{H}_{21}$ requires 141.1643.

tert-Butyl mesityl carbonate (1e). ${ }^{25}$ Colourless oil; yield: 48\%; $R_{\mathrm{f}} 0.96$ (hexane/ethyl acetate: 9/1); v (film) $1755(\mathrm{C}=\mathrm{O}), 1608,1486(\mathrm{HC}=\mathrm{C}), 1279 \mathrm{~cm}^{-1}(\mathrm{CO}) ; \delta_{\mathrm{H}} 1.55\left(9 \mathrm{H}, \mathrm{s}, \mathrm{Me}_{3} \mathrm{C}\right), 2.16$, $2.25(6 \mathrm{H}$ and $3 \mathrm{H}$, respectively, $2 \mathrm{~s}, 3 \times M e \mathrm{Ar}), 6.85(2 \mathrm{H}, \mathrm{s}, \mathrm{ArH}) ; \delta_{\mathrm{C}} 16.0(2 \mathrm{C}), 20.7(3 \times M e \mathrm{Ar})$, 27.3 (3C, $\left.\mathrm{Me}_{3} \mathrm{C}\right), 82.9$ (CO), 129.2 (2C), 129.8 (2C), 135.2, 146.1 (ArC), 151.5 (C=O); m/z 236 $\left(\mathrm{M}^{+},<1 \%\right), 177$ (16), 137 (11), 136 (100), 135 (19), 121 (36), 119 (18), 91 (21), 57 (74).

$\mathrm{N}$-(tert-Butoxycarbonyl)dioctylamine (1f). ${ }^{26}$ Colourless oil; yield: $62 \% ; R_{\mathrm{f}} 0.78$ (hexane/ethyl acetate: 9/1); $v$ (film) $1697(\mathrm{C}=\mathrm{O}), 1172 \mathrm{~cm}^{-1}(\mathrm{CO}) ; \delta_{\mathrm{H}} 0.78-0.98\left(6 \mathrm{H}, \mathrm{m}, 2 \times M e \mathrm{CH}_{2}\right), 1.17-1.37$, 1.41-1.59 [20H and $4 \mathrm{H}$, respectively, $\left.2 \mathrm{~m}, 2 \times \mathrm{Me}\left(\mathrm{CH}_{2}\right)_{6}\right], 1.45\left(9 \mathrm{H}, \mathrm{s}, \mathrm{Me}_{3} \mathrm{C}\right), 3.02-3.24(4 \mathrm{H}, \mathrm{m}$, $\left.2 \times \mathrm{CH}_{2} \mathrm{~N}\right) ; \delta_{\mathrm{C}} 14.0\left(2 \mathrm{C}, 2 \times \mathrm{MeCH}_{2}\right), 22.6(2 \mathrm{C}), 26.9(2 \mathrm{C}), 29.2(2 \mathrm{C}), 29.3(2 \mathrm{C}), 31.8(4 \mathrm{C}), 28.4$ 
$\left(3 \mathrm{C}, \mathrm{Me}_{3} \mathrm{C}\right), 47.0(2 \mathrm{C}, 2 \times \mathrm{CN}), 78.8(\mathrm{CO}), 155.6(\mathrm{C}=\mathrm{O}) ; \mathrm{m} / z 341\left(\mathrm{M}^{+}, 4 \%\right), 187$ (12), 186 (100), 174 (10), 142 (89), 88 (37), 57 (50).

4-Benzyl- $N$-(tert-butoxycarbonyl)piperidine (1g). Colourless oil; yield: $63 \% ; \quad R_{\mathrm{f}} 0.72$ (hexane/ethyl acetate: 9/1); $v$ (film) 3093, 3060, 3033, $1600(\mathrm{HC}=\mathrm{C}), 1693(\mathrm{C}=\mathrm{O}), 1168 \mathrm{~cm}^{-1}$ $(\mathrm{CO}) ; \delta_{\mathrm{H}} 1.00-1.27,1.53-1.79\left(2 \mathrm{H}\right.$ and $3 \mathrm{H}$, respectively, $2 \mathrm{~m}, 2 \times \mathrm{CH}_{2} \mathrm{CN}$ and $\left.\mathrm{CH}\right), 1.44(9 \mathrm{H}, \mathrm{s}$, $\left.\mathrm{Me}_{3} \mathrm{C}\right), 2.55\left(2 \mathrm{H}, \mathrm{d}, J=5.6 \mathrm{~Hz}, \mathrm{PhCH}_{2}\right), 2.39-2.84,3.79-4.25\left(2 \mathrm{H}\right.$ each, $\left.2 \mathrm{~m}, 2 \times \mathrm{CH}_{2} \mathrm{~N}\right), 6.94-7.35$ $(5 \mathrm{H}, \mathrm{m}, \mathrm{ArH}) ; \delta_{\mathrm{C}} 28.4\left(3 \mathrm{C}, \mathrm{Me}_{3} \mathrm{C}\right), 29.0\left(2 \mathrm{C}, 2 \times \mathrm{CH}_{2} \mathrm{CN}\right), 31.9\left(\mathrm{CHCH}_{2}\right), 38.1\left(\mathrm{CH}_{2} \mathrm{Ph}\right), 43.1$ $(2 \mathrm{C}, 2 \times \mathrm{CN}), 79.1(\mathrm{CO}), 125.9,128.2(2 \mathrm{C}), 129.0(2 \mathrm{C}), 140.2(\mathrm{ArC}), 154.8(\mathrm{C}=\mathrm{O}) ; \mathrm{m} / z 275\left(\mathrm{M}^{+}\right.$, 4\%), 220 (25), 219 (100), 216 (10), 202 (28), 175 (18), 174 (30), 158 (13), 130 (15), 129 (14), 128 (14), 126 (17), 113 (21), 91 (42), 84 (12), 69 (15), 57 (75), 56 (12); HRMS: M+ found 275.1885. $\mathrm{C}_{17} \mathrm{H}_{25} \mathrm{NO}_{2}$ requires 275.1885.

$\boldsymbol{N}$-(tert-Butoxycarbonyl)- $\boldsymbol{N}$-methylaniline $\quad(\mathbf{1 h}){ }^{27}$ Colourless oil; yield: $33 \% ; \quad R_{\mathrm{f}} \quad 0.70$ (hexane/ethyl acetate: 9/1); $v$ (film) 3077, 3028, $1598(\mathrm{HC}=\mathrm{C}), 1701(\mathrm{C}=\mathrm{O}), 1154 \mathrm{~cm}^{-1}(\mathrm{CO}) ; \delta_{\mathrm{H}}$ $1.45\left(9 \mathrm{H}, \mathrm{s}, \mathrm{Me}_{3} \mathrm{C}\right), 3.26(3 \mathrm{H}, \mathrm{s}, \mathrm{MeN}), 7.12-7.40(5 \mathrm{H}, \mathrm{m}, \mathrm{ArH}) ; \delta_{\mathrm{C}} 28.3\left(3 \mathrm{C}, \mathrm{Me}_{3} \mathrm{C}\right), 37.3$ $(\mathrm{MeN}), 80.2(\mathrm{CO}), 125.5(2 \mathrm{C}), 128.5(2 \mathrm{C}), 129.1,143.8(\mathrm{ArC}), 154.8(\mathrm{C}=\mathrm{O}) ; \mathrm{m} / \mathrm{z} 207\left(\mathrm{M}^{+}\right.$, 10\%), 152 (10), 151 (100), 134 (30), 107 (75), 106 (56), 77 (28), 57 (79).

O-tert-Butyl $\boldsymbol{S}$-(1-decyl) thiocarbonate (1i). Colourless oil; yield: 42\%; $R_{\mathrm{f}} 0.92$ (hexane/ethyl acetate: 9/1); $v$ (film) $1704(\mathrm{C}=\mathrm{O}), 1128(\mathrm{CO}), 841 \mathrm{~cm}^{-1}(\mathrm{CS}) ; \delta_{\mathrm{H}} 0.88\left(3 \mathrm{H}, \mathrm{t}, J=6.4 \mathrm{~Hz}, \mathrm{MeCH}_{2}\right)$, 1.14-1.42, 1.53-1.69 [14H and 2H, respectively, $\left.2 \mathrm{~m}, \mathrm{Me}\left(\mathrm{CH}_{2}\right)_{8}\right], 1.49\left(9 \mathrm{H}, \mathrm{s}, \mathrm{Me}_{3} \mathrm{C}\right), 2.78(2 \mathrm{H}, \mathrm{t}$, $\left.J=7.3 \mathrm{~Hz}, \mathrm{CH}_{2} \mathrm{~S}\right) ; \delta_{\mathrm{C}} 14.1\left(\mathrm{MeCH}_{2}\right), 22.7,29.1,29.3,29.5(2 \mathrm{C}), 29.9,30.9,31.8\left[\mathrm{Me}\left(\mathrm{CH}_{2}\right)_{8}\right]$, 28.2 (3C, $\left.\mathrm{Me}_{3} \mathrm{C}\right), 28.8$ (CS), 84.3 (CO) $169.6(\mathrm{C}=\mathrm{O}) ; \mathrm{m} / z 219\left(\mathrm{M}^{+}-57,<1 \%\right), 57$ (100), 56 (12), 55 (14); HRMS: $\mathrm{M}^{+}-101$, found 173.1365. $\mathrm{C}_{10} \mathrm{H}_{21} \mathrm{~S}$ requires 173.1364.

$\boldsymbol{O}$-tert-Butyl $\boldsymbol{S}$-cyclohexyl thiocarbonate $(\mathbf{1 j}) .{ }^{28}$ Colourless oil; yield: $75 \% ; R_{\mathrm{f}} 0.63$ (hexane); $v$ (film) $1701(\mathrm{C}=\mathrm{O}), 1129(\mathrm{CO}), 841 \mathrm{~cm}^{-1}(\mathrm{CS}) ; \delta_{\mathrm{H}} 1.10-2.11\left(10 \mathrm{H}, \mathrm{m}, 5 \times \mathrm{CH}_{2}\right), 1.50(9 \mathrm{H}, \mathrm{s}$, $\left.\mathrm{Me}_{3} \mathrm{C}\right), 3.14-3.36(1 \mathrm{H}, \mathrm{m}, \mathrm{CH}) ; \delta_{\mathrm{C}} 25.9(2 \mathrm{C}), 26.0,33.7(2 \mathrm{C})\left(5 \times \mathrm{CH}_{2}\right), 28.6\left(3 \mathrm{C}, \mathrm{Me}_{3} \mathrm{C}\right), 44.6$ (CS), $84.6(\mathrm{CO}), 169.6(\mathrm{C}=\mathrm{O}) ; m / z 216\left(\mathrm{M}^{+},<1 \%\right), 57(100)$.

$\boldsymbol{O}$-tert-Butyl $\boldsymbol{S}$-Phenyl thiocarbonate $(\mathbf{1 k}){ }^{24}$ Colourless oil; yield: $80 \% ; R_{\mathrm{f}} 0.64$ (hexane); $v$ (film) 3077, 3066, $1578(\mathrm{HC}=\mathrm{C}), 1727(\mathrm{C}=\mathrm{O}), 1124(\mathrm{CO}), 838 \mathrm{~cm}^{-1}(\mathrm{CS}) ; \delta_{\mathrm{H}} 1.51(9 \mathrm{H}, \mathrm{s}$, $\left.\mathrm{Me}_{3} \mathrm{C}\right), 7.34-7.45,7.47-7.57(3 \mathrm{H}$ and $2 \mathrm{H}$, respectively, $2 \mathrm{~m}, \mathrm{ArH}) ; \delta_{\mathrm{C}} 28.1\left(3 \mathrm{C}, \mathrm{Me}_{3} \mathrm{C}\right), 85.5$ (CO), 127.1, 129.1, 129.3 (2C), 134.8 (2C) (ArC), $167.9(\mathrm{C}=\mathrm{O}) ; m / z 210\left(\mathrm{M}^{+},<1 \%\right), 137$ (13), 110 (36), 109 (40), 65 (13), 57 (100).

\section{DTBB-catalysed lithiation of compounds 1. Preparation of products 2 . General procedure}

A solution of the protected substrate $1(1.0 \mathrm{mmol})$ in THF $(2 \mathrm{~mL})$ was dropwise added to a green suspension of lithium powder (63 mg, $9.0 \mathrm{mmol}$ ) and 4,4'-di-tert-butylbiphenyl (DTBB, $27 \mathrm{mg}$, $0.1 \mathrm{mmol})$ in THF $(5 \mathrm{~mL})$, under $\mathrm{Ar}$, at $0^{\circ} \mathrm{C}$. After stirring at the same temperature for the time indicated in Table 1, methanol $(5 \mathrm{~mL})$ was carefully added, the cooling bath was removed and the reaction was stirred till it reached room temperature. The yields of the deprotected products (Table 1, entries 1, 3-5, 7, 9, 10, 12 and 14-16) were determined by quantitative GC. Commercially available alcohols, amines or thiols $\mathbf{2}, n$-dodecane (internal standard) and $n$ hexadecane (internal standard for $\mathbf{2 e}$ ) were used in the determination of response factors. 
Compounds 2 (commercially available) were characterised by comparison of their physical and spectroscopic data (GC-MS) with authentic samples.

Isolation of the deprotected products $2 \mathbf{d}$ and $2 \mathrm{e}$. The lithiations of substrates $\mathbf{1 d}$ and $\mathbf{1 e}$ were repeated as indicated in the general procedure, but stirring of the reaction mixture was continued for $7 \mathrm{~h}$ at $0^{\circ} \mathrm{C}$ and for additional $13 \mathrm{~h}$ (for $\mathbf{1 d}$ ) or $17 \mathrm{~h}$ (for 1e) at room temperature. After cooling the reaction flasks at $0^{\circ} \mathrm{C}$, water $(5 \mathrm{~mL})$ was carefully added, the cooling bath was removed and the reactions were stirred till they reached room temperature again. The reactions were then extracted with ethyl acetate $(3 \times 20 \mathrm{~mL})$ and the organic layers were dried $\left(\mathrm{Na}_{2} \mathrm{SO}_{4}\right)$. The yields of the deprotected products (Table 1, entries 6 and 8) were estimated by quantitative GC, using commercially available alcohol $\mathbf{2 d}$, phenol $\mathbf{2 e}, n$-dodecane (internal standard for $\mathbf{2 d}$ ) and $n$ hexadecane (internal standard for $\mathbf{2 e}$ ) in the determination of response factors. After evaporation of the solvents (15 Torr) the resulting residues were purified by column chromatography (silica gel, hexane/ethyl acetate) to give pure alcohol $\mathbf{2 d}$ and phenol $\mathbf{2 e}$ in 42 and 30\% yield, respectively. Products 2d and 2e (commercially available) were fully characterised by comparison of their physical and spectroscopic data with authentic samples.

Isolation of the deprotected amines $\mathbf{2 g}$ and $\mathbf{2 h}$. The lithiations of carbamates $\mathbf{1 g}$ and $\mathbf{1 h}$ were repeated as indicated in the general procedure. Water $(5 \mathrm{~mL})$ was carefully added instead of methanol, the cooling bath was removed and the reactions were stirred till they reached room temperature. The reactions were then extracted with ethyl acetate $(3 \times 20 \mathrm{~mL})$ and the yields of the deprotected products (Table 1, entries 11 and 13) were estimated by quantitative GC, using commercially available amines $\mathbf{2 g}-\mathbf{h}$ and $n$-dodecane (internal standard) in the determination of response factors. The reaction mixtures were then acidified with $2 \mathrm{M} \mathrm{HCl}$ and the organic layers were discarded. The acidic aqueous phases were treated with a $\mathrm{NH}_{4} \mathrm{Cl} / \mathrm{NH}_{3}$ buffer solution until the $\mathrm{pH}$ was basic, extracted with ethyl acetate $(3 \times 20 \mathrm{~mL})$ and dried $\left(\mathrm{Na}_{2} \mathrm{SO}_{4}\right)$. Evaporation of the solvents (15 Torr) gave the pure amines $\mathbf{2 g}$ and $\mathbf{2 h}$ in 81 and $49 \%$ yield, respectively. Products $\mathbf{2 g}$ and $\mathbf{2 h}$ (commercially available) were fully characterised by comparison of their physical and spectroscopic data with authentic samples.

DTBB-catalysed lithiation of carbonate 1 a on a $10 \mathrm{mmol}$ scale. Isolation of the deprotected alcohol 2a. A solution of carbonate $1 \mathrm{a}(2.6 \mathrm{~g}, 10.0 \mathrm{mmol})$ in THF $(10 \mathrm{~mL})$ was dropwise added to a green suspension of lithium powder $(280 \mathrm{mg}, 40.0 \mathrm{mmol})$ and DTBB $(133 \mathrm{mg}, 0.5 \mathrm{mmol})$ in THF $(20 \mathrm{~mL})$, under Ar, at $0{ }^{\circ} \mathrm{C}$. After stirring at the same temperature for $5 \mathrm{~h}$, the excess of lithium powder was removed by filtration under $\mathrm{Ar}$ and water $(5 \mathrm{~mL})$ was carefully added to the filtrate at room temperature. The reaction was then extracted with ethyl acetate $(3 \times 20 \mathrm{~mL})$ and the combined organic layers were dried $\left(\mathrm{Na}_{2} \mathrm{SO}_{4}\right)$. After evaporation of the solvents (15 Torr) the resulting residue was purified by column chromatography (silica gel, hexane/ethyl acetate) giving pure alcohol 2a in $82 \%$ yield. Compound 2a (commercially available) was fully characterised by comparison of its physical and spectroscopic data with an authentic sample. 


\section{Acknowledgements}

This work was generously supported by the Dirección General de Enseñanza Superior (DGES) of the current Spanish Ministerio de Educación y Ciencia (MEC; grant no. CTQ2004-01261) and the Generalitat Valenciana (grant no. GRUPOS03/135). R. A. thanks the Spanish Ministerio de Educación y Ciencia for a predoctoral fellowship. C. B. thanks the University of Alicante for a predoctoral fellowship. We also thank MEDALCHEMY S.L. for a gift of chemicals.

\section{References}

1. (a) Carpino, L. A. J. Am. Chem. Soc. 1957, 79, 98. (b) For a review, see: Carpino, L. A. Acc. Chem. Res. 1973, 6, 191.

2. Kunz, H.; Waldmann, H. In Comprehensive Organic Synthesis; Trost, B. M.; Fleming, I.; Winterfeldt, E., Eds.; Pergamon Press: Oxford, 1991; Vol. 6; Chapter 3.1.

3. Greene, T. W.; Wuts, P. G. M. Protective Groups in Organic Synthesis; 3rd Ed.; John Wiley \& Sons: New York, 1999; pp 281, 484-485, 518-524.

4. Kocienski, P. J. Protecting Groups; Thieme: Stuttgart, 2004; pp 341-343, 378, 505-511.

5. Fujii, N.; Otaka, A.; Ikemura, O.; Akaji, K.; Funakoshi, S.; Hayashi, Y.; Kuroda, Y.; Yajima, H. J. Chem. Soc., Chem. Commun. 1987, 274.

6. Sakaitani, M.; Ohfune, Y. J. Org. Chem. 1990, 55, 870.

7. Evans, E. F.; Lewis, N. J.; Kapfer, I.; Macdonald, G.; Taylor, R. J. K. Synth. Commun. 1997, $27,1819$.

8. (a) Routier, S.; Saugé, L.; Ayerbe, N.; Coudert, G.; Mérour, J.-Y. Tetrahedron Lett. 2002, 43, 589. (b) Jacquemard, U.; Bénéteau, V.; Lefoix, M.; Routier, S.; Mérour, J.-Y.; Coudert, G. Tetrahedron 2004, 60, 10039.

9. Nigam, S. C.; Mann, A.; Taddei, M.; Wermuth, C.-G. Synth. Commun. 1989, 19, 3139.

10. Hwu, J. R.; Jain, M. L.; Tsay, S.-C.; Hakimelahi, G. H. Tetrahedron Lett. 1996, 37, 2035.

11. For reviews, see: (a) Yus, M. Chem. Soc. Rev. 1996, 25, 155. (b) Ramón, D. J.; Yus, M. Eur. J. Org. Chem. 2000, 225. (c) Yus, M. Synlett 2001, 1197. (d) Yus, M.; Ramón, D. J. Latv. J. Chem. 2002, 79. (e) Ramón, D. J.; Yus, M. Rev. Cubana Quim. 2002, 14, 76. (f) Yus, M. In The Chemistry of Organolithium Compounds; Rappoport, Z.; Mareck, I., Eds.; J. Wiley \& Sons: Chichester, 2004; Chapter 11.

12. For mechanistic studies, see: (a) Yus, M.; Herrera, R. P.; Guijarro, A. Tetrahedron Lett. 2001, 42, 3455. (b) Yus, M.; Herrera, R. P.; Guijarro, A. Chem. Eur. J. 2002, 8, 2574. (c) Herrera, R. P.; Guijarro, A.; Yus, M. Tetrahedron Lett. 2003, 44, 1309.

13. For a polymer version of this reaction, see: (a) Gómez, C.; Ruiz, S.; Yus, M. Tetrahedron Lett. 1998, 39, 1397. (b) Gómez, C.; Ruiz, S.; Yus, M. Tetrahedron 1999, 55, 7017. (c) Yus, M.; Gómez, C.; Candela, P. Tetrahedron 2002, 58, 6207. (d) Candela, P.; Gómez, C.; Yus, M. Russ. J. Org. Chem. 2004, 40, 795. 
14. This methodology has been extensively used in our laboratories for the following applications: generation of organolithium compounds from non-halogenated materials, ${ }^{14 a}$ preparation of very sensitive functionalised organolithium compounds, ${ }^{14 \mathrm{~b}-\mathrm{g}}$ reductive ringopening of heterocycles, ${ }^{14 \mathrm{~h}-\mathrm{j}}$ generation of polylithium synthons ${ }^{14 \mathrm{k}, \mathrm{l}}$ and for the activation of other metals, ${ }^{14 \mathrm{~m}}$ especially nickel. ${ }^{14 \mathrm{n}, \mathrm{o}}$ For reviews from our group, see: (a) Guijarro, D.; Yus, M. Recent Res. Dev. Org. Chem. 1998, 2, 713. (b) Nájera, C.; Yus, M. Trends Org. Chem. 1991, 2, 155. (c) Nájera, C.; Yus, M. Recent Res. Dev. Org. Chem. 1997, 1, 67. (d) Nájera, C.; Yus, M. Curr. Org. Chem. 2003, 7, 867. (e) Nájera, C.; Sansano, J. M.; Yus, M. Tetrahedron 2003, 59, 9255. (f) Chinchilla, R.; Nájera, C.; Yus, M. Chem. Rev. 2004, 104, 2667. (g) See also the especial issue of Tetrahedron Symposium in Print devoted to "Functionalised Organolithium Compounds" Nájera, C.; Yus, M., Guest Eds., Tetrahedron 2005, 61, 3125. (h) Yus, M.; Foubelo, F. Rev. Heteroatom. Chem. 1997, 17, 73. (i) Yus, M.; Foubelo, F. Targets Heterocycl. Syst. 2002, 6, 136. (j) Yus, M. Pure Appl. Chem. 2003, 75, 1453. (k) Foubelo, F.; Yus, M. Trends Org. Chem. 1998, 7, 1. (1) Foubelo, F.; Yus, M. Curr. Org. Chem. 2005, 9, 459. (m) Guijarro, A.; Gómez, C.; Yus, M. Trends Org. Chem. 2000, 8, 65. (n) Alonso, F.; Radivoy, G.; Yus, M. Russ. Chem. Bull. 2003, 52, 2563. (o) Alonso, F.; Yus, M. Chem. Soc. Rev. 2004, 33, 284.

15. Yus, M.; Behloul, C.; Guijarro, D. Synthesis 2003, 2179.

16. Behloul, C.; Guijarro, D.; Yus, M. Synthesis 2004, 1274.

17. Behloul, C.; Guijarro, D.; Yus, M. Tetrahedron 2005, 61, 6908.

18. Behloul, C.; Guijarro, D.; Yus, M. Tetrahedron 2005, 61, 9319.

19. Behloul, C.; Guijarro, D.; Yus, M. Synthesis 2006, 309.

20. Part of this work has been preliminary communicated at the $9^{\text {th }}$ International Electronic Conference on Synthetic Organic Chemistry (ECSOC-9), communication nº A009.

21. Yus, M.; Martínez, P.; Guijarro, D. Tetrahedron 2001, 57, 10119.

22. Watson, S. C.; Eastham, J. F. J. Organomet. Chem. 1967, 9, 165.

23. Matisova-Rychla, L.; Rychly, J.; Pastusakova, V.; Schulz, M. Chem. Pap. 1986, 40, 61; Chem. Abstr. 1986, 104, 225267.

24. Houlihan, F.; Bouchard, F.; Frechet, J. M. J.; Willson, C. G. Can. J. Chem. 1985, 63, 153.

25. Iwasaki, F. Jpn. Kokai Tokkyo Koho 1994, JP 06128198; Chem. Abstr. 1994, 122, 80875.

26. Kobayashi, E.; Numata, J.; Yamachika, M.; Yamamoto, M. Eur. Pat. Appl. 2001, EP 1164433; Chem. Abstr. 2001, 136, 45682.

27. Bartoli, G.; Bosco, M.; Locatelli, M.; Marcantoni, E.; Massaccesi, M.; Melchiorre, P.; Sambri, L. Synlett 2004, 1794.

28. Kawabata, J.; Iwasaki, F.; Koyanagi, S. Jpn. Kokai Tokkyo Koho 1995, JP 07025831; Chem. Abstr. 1995, 123, 8811. 